\title{
FastCloning: a highly simplified, purification-free, sequence- and ligation-independent PCR cloning method
}

\author{
Chaokun Li', Aiyun Wen', Benchang Shen ${ }^{1,3}$, Jia Lu², Yao Huang ${ }^{2}$ and Yongchang Chang ${ }^{\text {* }}$
}

\begin{abstract}
Background: Although a variety of methods and expensive kits are available, molecular cloning can be a timeconsuming and frustrating process.

Results: Here we report a highly simplified, reliable, and efficient PCR-based cloning technique to insert any DNA fragment into a plasmid vector or into a gene (CDNA) in a vector at any desired position. With this method, the vector and insert are PCR amplified separately, with only 18 cycles, using a high fidelity DNA polymerase. The amplified insert has the ends with 16-base overlapping with the ends of the amplified vector. After Dpnl digestion of the mixture of the amplified vector and insert to eliminate the DNA templates used in PCR reactions, the mixture is directly transformed into competent E. coli cells to obtain the desired clones. This technique has many advantages over other cloning methods. First, it does not need gel purification of the PCR product or linearized vector. Second, there is no need of any cloning kit or specialized enzyme for cloning. Furthermore, with reduced number of PCR cycles, it also decreases the chance of random mutations. In addition, this method is highly effective and reproducible. Finally, since this cloning method is also sequence independent, we demonstrated that it can be used for chimera construction, insertion, and multiple mutations spanning a stretch of DNA up to $120 \mathrm{bp}$.

Conclusion: Our FastCloning technique provides a very simple, effective, reliable, and versatile tool for molecular cloning, chimera construction, insertion of any DNA sequences of interest and also for multiple mutations in a short stretch of a cDNA.
\end{abstract}

\section{Background}

Molecular cloning is one of the most widely used techniques in biomedical research laboratories. Traditionally, molecular cloning joins insert and vector by T4 DNA ligase after restriction digestion to excise insert from a donor vector or from a PCR product with restriction enzyme recognition sites added to the ends [1]. Although this is a widely used method, it involves multiple steps and is time consuming. This multi-step process also makes it difficult or complicated for troubleshooting. To overcome the difficulties encountered in the original cloning method, many other alternative cloning methods have been developed over the last two decades. These methods include

\footnotetext{
* Correspondence: yongchang.chang@chw.edu

'Division of Neurobiology, Barrow Neurological Institute, St. Joseph's Hospital and Medical Center, Phoenix, AZ, USA

Full list of author information is available at the end of the article
}

TA cloning [2], ligation independent cloning with T4 DNA polymerase [3,4], GATEWAY recombinational cloning [5], and more recent sequence- and ligation-independent cloning kits, such as CloneEZ (GenScript USA Inc., Piscataway, NJ, USA), one step cloning [6], and overlap extension PCR cloning [7]. However, each of these techniques has its own limitations. For example, TA cloning uses regular Taq DNA polymerase to add a single 3'-A overhang to the ends of the PCR product. The PCR product is directly cloned into a TA cloning vector with a complementary 3 '- $T$ overhang in both ends without restriction digestion. The limitations of this method are low fidelity of Taq DNA polymerase causing unwanted mutations and requirement of subcloning into the final target vector with restriction digestion and ligation. The early ligation independent cloning uses the 3 '-exonucnease activity of T4 DNA polymerase to create 15 -base 
5 'overhangs in the ends of insert and complementary 5 ' overhangs in the ends of vector. This technique requires specific sequences to create 15-base overhangs. Gateway recombinational cloning uses site-specific recombination to transfer cDNAs between donor and destination vectors, which requires additional specific sequences for recombination. The latest ligation-independent cloning, such as CloneEZ and In-Fusion cloning kits, uses some DNA polymerase to generate sticky ends in the vector and insert without specific sequence requirement, except for restriction sites to linearize the vector. However, the new ligation independent cloning still requires purification of the digested vector and PCR-amplified insert, and the purchase of purification and cloning kits. Similarly, overlap extension PCR cloning also requires purification of the first round PCR products (vector and insert) and an additional round overlap extension PCR, which usually generates multiple bands, for producing linked vector and insert. One-step "quick assemble" cloning does not need purification of PCR products. However, it includes two sequential 35-cycle PCRs with a total number of 70 cycles. The first-round PCRs are used to amplify insert and linear vector. The second-round PCR is essentially the overlap extension PCR to assemble vector and insert into a single linear PCR product. Another ligation independent cloning technique, using nick DNA endonuclease to create long single-strand 5' overhangs in the vector and PCR-amplified insert [8], requires specific sequences for nick DNA endonuclease and purification of the PCR product.

Purification of PCR product not only takes extra time and requires purification kit, but also potentially creates additional problems. For example, to compensate for the loss of PCR product during purification, the number of PCR cycles is generally 25-30. High number of PCR cycles increases the chance of random mutations or, in our experiences, dramatically decreases cloning efficiency for the PCR products generated by high fidelity DNA polymerases. This is especially true for the enzyme with high processability, such as pfuUltraII DNA polymerase. The decrease in cloning efficiency cannot be completely overcome by using state-of-the-art cloning kits, such as CloneEZ. Using this kit (with gel purification of digested vectors and PCR-amplified inserts), the successful rate in our laboratory still varies significantly with overall successful rate less than 50\%. In the PCR-based QuickChange mutagenesis, the number of PCR cycles is recommended to be 12-18. Increase in PCR cycles could decrease efficiency according to the QuickChange protocol. Thus, these phenomena made us believe that the proofreading PCR enzymes could potentially damage the ends of PCR products if the number of PCR cycles increases. This could be due to the fact that the high fidelity DNA polymerases with the proofreading ability have 3' exonuclease activity. In the presence of dNTPs, the ends of PCR products can be protected from this 3' exonuclease activity. It is possible that depletion of dNTPs in high number of PCR cycles or dilution of dNTPs in the early stage of purification would weaken the 3' ends protection against 3 ' exonuclease activity of the DNA polymerase, which results in the damage of PCR products and makes cloning extremely difficult.

To circumvent the above-mentioned problems, we developed a cloning method termed FastCloning. With this method, both insert and vector are amplified by 18 PCR cycles with a high fidelity DNA polymerase. The unpurified PCR products of the vector and insert are then directly mixed at some ratios (see below) and digested by $D p n I$ restriction enzyme to destroy their methylated DNA templates. Finally, the digested mixture is transformed into competent cells to obtain the target clones. The PCR amplification of vector is designed to make it possible to clone an insert into any position of the vector, to bypass vector digestion (and restriction site limitation) and purification steps, and to be compatible with $D p n I$ digestion of the insert without further inactivation of the enzyme. Thus, this method can be used to construct cDNAs of fusion proteins or chimera without limitation by available restriction sites. In this study, we experimentally validated all these applications with our new method. In addition, a similar method can be easily adapted for deletion of a DNA fragment.

\section{Methods}

Figure 1 illustrates the highly simplified procedure of our FastCloning method. Briefly, after gel confirmation of PCR products, the remaining unpurified PCR reactions, containing amplified vector and insert, are mixed and digested with $D p n I$ for 1 hour at $37^{\circ} \mathrm{C}$. The digested mixture is then directly transformed into chemically competent Stratagene XL-10 Gold or NEB 10-beta E. coli cells.

The primers were designed with Oligo Analyzer 1.5 (http://www.genelink.com) to have an annealing temperature around $60^{\circ} \mathrm{C}$ (Nearest Neighbor method). The forward primer for vector amplification is in the 3 ' side of the polylinker region. The reverse primer for vector amplification is in the 5 ' side of the polylinker, and its reverse and complementary sequence was generated by Oligo Explorer 1.5. The primers for insert amplification have insert-specific sequences and additional 15-17 bases (depending on the GC content) overlapping with the vector ends. The specific sequences of primers used in this study are listed in Table 1. All the primers used in this study were synthesized by Invitrogen Corporation (Carlsbad, CA).

The PCR reaction components were: $50 \mu \mathrm{l}$ total volume, $0.5 \mu$ l Phusion DNA polymerase (New England Biolabs, Ipswich, MA), or $0.8 \mu \mathrm{l} P f u$ Turbo, or PfuUltra DNA polymerase (Agilent Technologies, Inc, Santa Clara, CA), $5 \mu \mathrm{l} 10 \times$ buffer; $5 \mu \mathrm{l}$ of $2.5 \mathrm{mM}$ dNTPs; 


\section{Step 1: PCR amplification of vector and insert}
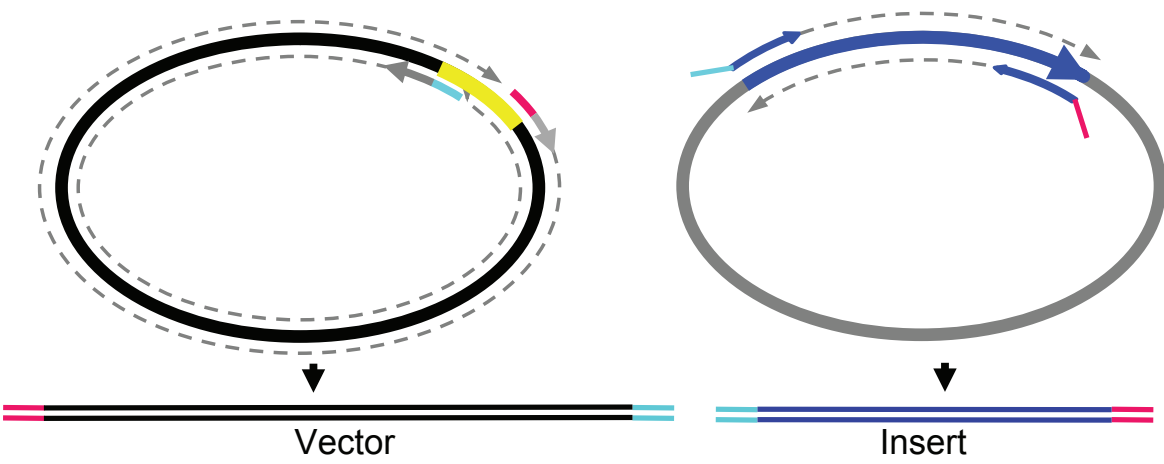

Step 2: Dpnl digestion of DNA templates

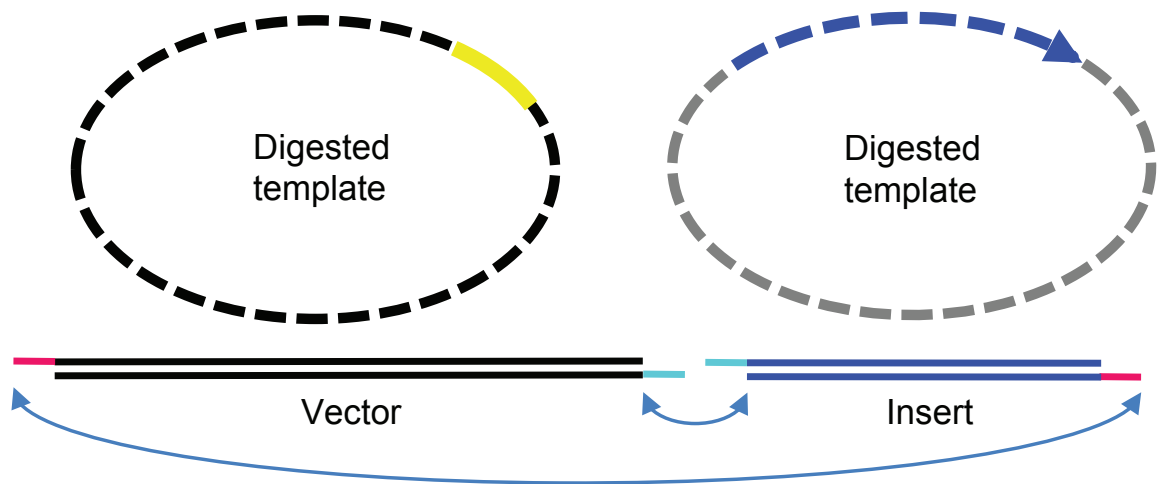

Step 3: Transformation into competent cells

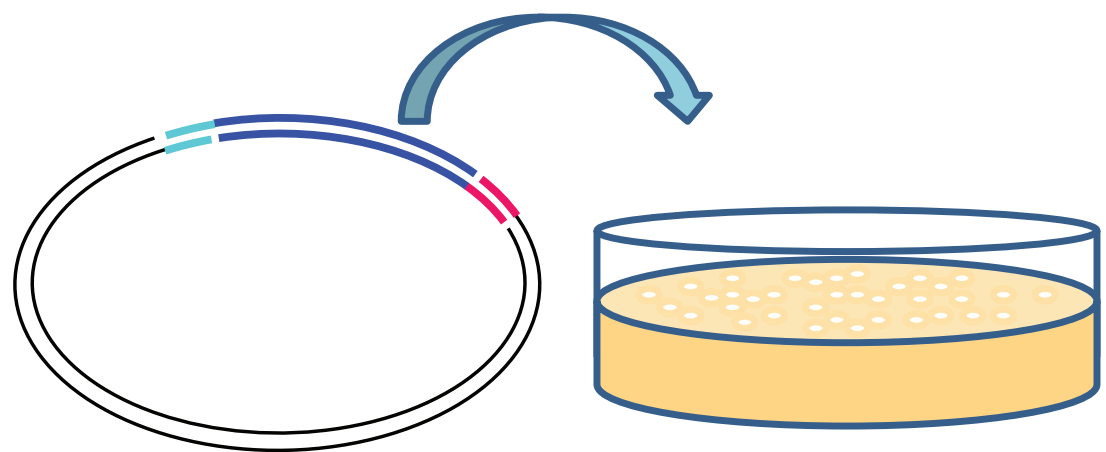

Figure 1 The procedures for FastCloning: Step 1. PCR amplification of vector and insert. Note that the primer pair for insert amplification has 16-base tails overlapping with the PCR-amplified vector ends. Step 2. Dpnl digestion. The parent DNA templates (if in a plasmid) for PCR amplification needs to be methylated in order to be compatible to Dpnl digestion. Although the detailed mechanism is not known, it is likely that the 3' exonuclease activity of the high fidelity DNA polymerase directly creates sticky ends for the overlapped regions of the vector and insert during Dpnl digestion, allowing them to form a circular construct with nicks. Step 3. transformation into competent $E$. coli. cells. The nicks will be repaired after transformation into the bacteria.

10 ng of plasmid DNA template; 5 pmol of each primer. The PCR cycling parameters were $98^{\circ} \mathrm{C} 3 \mathrm{~min},\left(98^{\circ} \mathrm{C} 10\right.$ sec, $\left.55^{\circ} \mathrm{C} 30 \mathrm{sec}, 72^{\circ} \mathrm{C} 20 \mathrm{sec} / \mathrm{kb}\right) \times 18$ cycles, $72^{\circ} \mathrm{C} 5$ min, and $4^{\circ} \mathrm{C}$ infinite for Phusion DNA polymerase, and $95^{\circ} \mathrm{C} 3 \mathrm{~min},\left(95^{\circ} \mathrm{C} 15 \mathrm{sec}, 55^{\circ} \mathrm{C} 1 \mathrm{~min}, 72^{\circ} \mathrm{C} 1 \mathrm{~min} / \mathrm{kb}\right) \times$
18 cycles, $72^{\circ} \mathrm{C} 5 \mathrm{~min}$, and $4^{\circ} \mathrm{C}$ infinite for $P f u$ Turbo or PfuUltra DNA Polymerase. The PCR products $(5 \mu \mathrm{l}$ for each product) were examined with $1 \%$ agarose gel electrophoresis with ethidium bromide staining using VWR Mini Gel electrophoresis setup (VWR International, 
Table 1 Primers used in the cloning or mutagenesis experiments

\begin{tabular}{|c|c|c|}
\hline Primer name & Primer description & Primer sequence \\
\hline pGEMHEStartRev & Reverse primer for pGEMHE vector with start codon & CATGGCCAAAGTTGAGCGTTTATTCTG \\
\hline pGEMHEStopFwd & Forward primer for pGEMHE vector with stop codon & TAAACCAGCCTCAAGAACACC \\
\hline p3X14startRV & Reverse primer for p3xFlag-cmv-14 vector with start codon & CATGGTGGCGAATTCGCGGCCGCAAGC \\
\hline p3X14endFW & Forward primer for p3xFlag-cmv-14 vector & GGATCCCGGGCTGACTAC \\
\hline CHRNA9Startez & Forward primer for CHRNA9 cloning into PGEMHE & CTCAACTTTGGCCATGAACTGGTCCCATTCCTGCAT \\
\hline CHRNA9Stopez & Reverse primer for CHRNA9 cloning into pGEMHE & TCTTGAGGCTGGTTTAATCCGCTCTTGCTATGATCAAA \\
\hline CHRNB2qk5 & Forward primer for CHRNB2 cloning into pGEMHE & GCTCAACTTTGGCCATGGCCCGGCGCTGCGG \\
\hline CHRNB2qk3 & Reverse primer for CHRNB2 cloning into pGEMHE & TTCTTGAGGCTGGTTTACTTGGAGCTGGGGGCTG \\
\hline CHRNA4_qk5 & Forward primer for CHRNA4 cloning into pGEMHE & GCTCAACTTTGGCCATGGAGCTAGGGGGCCC \\
\hline CHRNA4_qk3 & Reverse primer for CHRNA4 cloning into pGEMHE & TTCTTGAGGCTGGTTTAGATCATGCCAGCCAGCC \\
\hline ECSM2_qk5 & Forward primer for ECSCR cloning into pGEMHE & GCTCAACTTTGGCCATGGGCACCGCAGGAGC \\
\hline ECSM2_qk3 & Reverse primer for ECSCR cloning into pGEMHE & TTCTTGAGGCTGGTTTAAAGAACCTTCTCTGCTGAGAG \\
\hline HEstartFW-p3X14 & Forward primer for ECSCR cloning into p3xFlag-cmv-14 & CGAATTCGCCACCATGGGCACCGCAGGAGC \\
\hline HEendRV-p3X14 & Reverse primer for ECSCR cloning into p3xFlag-cmv-14 & AGTCAGCCCGGGATCCAAGAACCTTCTCTGCTGAGAG \\
\hline ZACNqk5 & Forward primer for ZACN cloning into pGEMHE & GCTCAACTTTGGCCATGATGGCCCTATGGTCCCTG \\
\hline ZACNqk3 & Reverse primer for ZACN cloning into pGEMHE & TTCTTGAGGCTGGTTTACAGTCTAGGCCGCCTGC \\
\hline APBB1qk5 & Forward primer for APBB1 cloning into pGEMHE & GCTCAACTITGGCCATGTCTGTTCCATCATCACTGAG \\
\hline APBB1qk3 & Reverse primer for APBB1 cloning into pGEMHE & TTCTTGAGGCTGGTTTATGGGGTATGGGCCCCCA \\
\hline HTR3A5P381_5A5 & Forward primer of HTR3A for 5 Pro mutated to Ala & CGCTGCCGCAGCAGCTCGGGAGGCCTCGCTG \\
\hline HTR3A5P381_5A3 & Reverse primer of HTR3A for 5 Pro mutated to Ala & GAGCTGCTGCGGCAGCGCTACATCTGTCCCTCGGG \\
\hline pLXSN3'fwd & Forward primer for pLXSN vector & GGATCCGGCTGTGGAATGTG \\
\hline pLXSN5'rev & Forward primer for pLXSN vector & GAATTCCGGCGCCTAGAGAA \\
\hline Akt3v1\&2Start & Forward primer for Akt3v1 \& v2 with Kozak sequence & CTAGGCGCCGGAATTCCATCATGAGCGATGTTACCATTGTG \\
\hline Akt3v1StopRev & Reverse primer for Akt3v1 cloning into pLXSN & TTCCACAGCCGGATCCTTATTCTCGTCCACTTGCAGAGTAG \\
\hline Akt3v2StopRev & Reverse primer for Akt3v2 cloning into pLXSN & TTCCACAGCCGGATCCTTATTITICCAGTTACCCAGCATGC \\
\hline$\beta 2 \beta 4 N \_R v$ & Reverse primer for vector along with $\mathrm{N}$-terminal cDNA & GATGATGAGGTTGATGGTGTAG \\
\hline$\beta 2 \beta 4 C_{-} F w$ & Forward primer for insert amplification & CATCAACCTCATCATCCCCTG \\
\hline$\beta 2$ StopRv_HE & Reverse primer for $C$-terminal $\beta 2$ insert amplification & CTTGAGGCTGGTITACTTGGAGCTGGGGGCTG \\
\hline$\beta 4$ StopRv_HE & Reverse primer for $C$-terminal $\beta 4$ insert amplification & CTTGAGGCTGGTTIAGTCACGCTGGGCAGCGT \\
\hline AChBPins1Fw & Forward primer for AChBP insert1 to $\alpha 7$ nAChR & $\begin{array}{l}\text { CCCGGCCCCACCAAGGACGACCCCCTGACCGTGACCCTGGG } \\
\text { CTTCACCCTGCAGGACATCATGGACGTGGATGAGAAGAAC }\end{array}$ \\
\hline AChBPins1Rv & Reverse primer for AChBP insert1 to $\alpha 7 \mathrm{nAChR}$ & $\begin{array}{l}\text { CTTGGTGGGGCCGGGGTACATGGGGCTTCTGTTGAACA } \\
\text { GGTCGCTCTTCAGTCTCATCTGGAACTCGCCTTGCAGG }\end{array}$ \\
\hline AChBPins2Fw & Forward primer for AChBP insert2 to $\alpha 7 \mathrm{nAChR}$ & $\begin{array}{l}\text { TGTGGGACCCCAACGAGTACGGCAACATCACCGACTTCAGA } \\
\text { ACCAGCGCCGCCGACATTTGGAAACCAGACATTCTTCTCTA }\end{array}$ \\
\hline AChBPins2Rv & Reverse primer for $\mathrm{AChBP}$ insert2 to $\alpha 7 \mathrm{nAChR}$ & $\begin{array}{l}\text { TCGTTGGGGTCCCACATCAGGCTGTTCAGCTTCCATCTCT } \\
\text { GCTGCTCGTAGTACACGGTGGTTAAAACTTGGTTCTTCTC }\end{array}$ \\
\hline AChBPins3Fw & Forward primer for AChBP insert3 to $\alpha 7 \mathrm{nAChR}$ & $\begin{array}{l}\text { TCGCCGTGGTGACCCACGACGGCAGCGTGATGTTCATCCCCG } \\
\text { CCCAGAGACTGAGCTTCATGTGCTACATCGATGTACGCTGG }\end{array}$ \\
\hline AChBPins3Rv & Reverse primer for AChBP insert3 to $\alpha 7 \mathrm{nAChR}$ & $\begin{array}{l}\text { TGGGTCACCACGGCGATCTGGGGGCTCAGCACCTGCACGGGTC } \\
\text { TGGTGCTGCTGTAGGCGGTAATGTCTGGTTTCCAAATGTCGG }\end{array}$ \\
\hline AChBPins4Fw & Forward primer for AChBP insert 4 to $\alpha 7 \mathrm{nAChR}$ & $\begin{array}{l}\text { CTTCGAGATCGACCTGAAGACCGACACCGA } \\
\text { CCAGGTGGATATCAGTGGCTATATCCCCA }\end{array}$ \\
\hline AChBPins4Rv & Reverse primer for AChBP insert 4 to $\alpha 7 \mathrm{nAChR}$ & $\begin{array}{l}\text { CAGGTCGATCTCGAAGCCGCTGTACACCCAGCTG } \\
\text { CCGAACTTCACGGCGCAGTGCTGCACATCAAAGG }\end{array}$ \\
\hline AChBPins5Fw & Forward primer for AChBP insert5 to $\alpha 7 \mathrm{nAChR}$ & $\begin{array}{l}\text { TACAGCTGCTGCCCCGAGCCCTACATCGACGTGAA } \\
\text { CCTGGTGGTGAAGTTCCGCCGCAGGACACTCTAC }\end{array}$ \\
\hline AChBPins5Rv & Reverse primer for AChBP insert 5 to $\alpha 7 \mathrm{nAChR}$ & $\begin{array}{l}\text { CGGGGCAGCAGCTGTAGTGCTGCACCTGTCTGGTCT } \\
\text { GGGTGGCGCTCAGGATGTCCCATTCTCCATTGGGGA }\end{array}$ \\
\hline
\end{tabular}

Marietta, GA, USA) running at $100 \mathrm{~V}$ for $30 \mathrm{~min}$. The PCR products were then visualized under a UV transilluminator, and gel pictures were taken using an AGFA scanner.
After confirmation of PCR products, $1 \mu \mathrm{l}$ of $D p n \mathrm{I}$ enzyme (New England Biolabs) was added into the remaining unpurified PCR reactions $(45 \mu \mathrm{l}$ for each product) for vector or insert separately. The vector and 
insert were then mixed with $1: 1$ ratio $(1: 1,1: 2$, and $1: 4$ for $\alpha 9 \mathrm{nAChR}$ subunit), and digested at $37^{\circ} \mathrm{C}$ for 1 hour. Two micro-liters (2, 4, and $8 \mu$ for $\alpha 9$ nAChR) of the digested vector-insert mixture were then added to $40 \mu \mathrm{l}$ of chemically competent XL-10 Gold E. coli cells (prepared with rubidium chloride method) unless indicated otherwise. The mixture was then incubated for $30 \mathrm{~min}$ on ice. After heat shock at $42^{\circ} \mathrm{C}$ for $45 \mathrm{sec}$, $350 \mu \mathrm{l}$ of SOC medium was added to the mixture. After 60 min shaking at $37^{\circ} \mathrm{C}$ and $350 \mathrm{rpm}$ with an Eppendorf Thermomixer, the entire content was plated onto the LB agar plate containing $100 \mu \mathrm{g} / \mathrm{ml}$ ampicillin. The plates were then incubated at $37^{\circ} \mathrm{C}$ overnight. Next day, colonies from each constructs were picked for PCR confirmation of each construct using GoTaq DNA polymerase (Promega, Madison, WI, USA) and vector specific primers, and also for inoculation in the LB medium (with ampicillin) for overnight culture of each clone for mini-prep. The DNA mini-prep was performed using QIAprep Spin Miniprep Kit (QIAGEN, Valencia, CA, USA). All the cloned sequences were finally confirmed by automated DNA sequencing at the DNA lab of the Arizona State University using primers in the vectors.

Figure 2 illustrates the application of this method to construct cDNAs encoding chimeric or fusion proteins. In this case, the PCR amplification of the vector also includes part of the cDNA in both ends. To insert a cDNA encoding a full-length protein, such as green fluorescence protein, the insert amplification should cover the entire coding region of the cDNA. For homologous domain swap chimera, insert amplification only needs to cover the corresponding region of a cDNA encoding a homologous fragment of protein. The detailed experimental procedures for chimera construction are essentially the same as cloning.

For insertion (or substitution) of a short DNA fragment $(<120 \mathrm{bp})$, the insert can be directly included in the primer sequences for vector amplification (Figure 3 ). This is a very convenient way to insert a short tag (such as myc tag or FLAG-tag) or to replace a short DNA fragment for chimera construction without limitation of the availability of specific DNA template for the insert. Introducing multiple mutations in a short (up to $120 \mathrm{bp}$ ) stretch of a cDNA is equivalent to replacing a short DNA fragment for chimera construction.

\section{Results and Discussion}

As a proof of principle, we subcloned several cDNAs into different vectors (human nicotinic receptor (nAChR) $\alpha 4, \alpha 9$ and $\beta 2$ subunits, and serotonin receptor type $3 \mathrm{~A}\left(5-\mathrm{HT}_{3} \mathrm{~A}\right)$ subunit into the pGEMHE vector, human endothelial cell-specific molecule 2 (ECSM2) into the p3XFLAG-CMV-14 vector, and Akt3v1 or Akt3v2 into pLXSN vector). pGEMHE is a vector containing 5' and 3' untranslated regions (5'UTR and 3'UTR) of Xenopus $\beta$-globin, which is highly expressed in Xenopus oocytes. With this vector, the protein expression level of the inserted gene in Xenopus oocyte can be increased by up to 200-fold [9].

We first optimized cloning conditions by cloning $\alpha 9$ $n A C h R$ subunit cDNA into the pGEMHE vector. Figure $4 \mathrm{~A}$ is an example of agarose gel electrophoresis for verification of PCR products of the vector ( $\mathrm{pGEMHE)} \mathrm{and}$ insert ( $\alpha 9 \mathrm{nAChR})$. In each lane, $5 \mu \mathrm{l}$ of PCR products was loaded. Note that amplification efficiency is the highest for Phusion DNA Polymerases and lowest for Pfu Turbo. We then compared cloning efficiency, in terms of number of colonies produced after transformation, for optimal ratio of vector and insert during $D p n \mathrm{I}$ enzyme digestion and optimal amount of the vector-insert mixture used in transformation. Vectors and inserts were mixed with three different ratios $(1: 1,1: 2$, and $1: 4$ with a total volume of $16 \mu \mathrm{l}$ ), and incubated at $37^{\circ} \mathrm{C}$ for $1 \mathrm{~h}$. Different amounts $(2,4$, and $8 \mu \mathrm{l})$ of the DpnI-digested mixtures were then added into $40 \mu \mathrm{l}$ of chemically competent XL-10 Gold E.coli cells for transformation. The number of colonies that grew on each plate was counted next day. Figure $4 \mathrm{~B}$ shows the number of colonies that resulted from different DNA polymerases, vector to insert ratios $(1: 1,1: 2$, and $1: 4)$, and the amount of vector-insert mixture $(2 \mu \mathrm{l}, 4 \mu \mathrm{l}$, and $8 \mu \mathrm{l})$ used for transformation. The results suggest that the best combination for colony formation is Phusion DNA polymerase amplification with 1:1 vector/insert ratio in $D p n I$ digestion and $2 \mu \mathrm{l}$ of vector-insert mix for transformation. Four colonies from the plates resulting from each DNA polymerase were picked up for DNA mini-prep. The PCR confirmation of the insert in the target vector (Figure $4 \mathrm{C}$ ) was performed with the GoTaq DNA polymerase (Promega Corporation, Madison, WI) using the pGEMHE vector specific primer pair. Our results indicated that > $90 \%$ colonies (11 out of 12 ) were positive. We have also compared colony production efficiency for three different numbers of PCR cycle $(12,18$, and 24 cycles) and three different durations $(1,2$, and 4 hours) of incubation time for $37^{\circ} \mathrm{C} \mathrm{DpnI} \mathrm{digestion} \mathrm{with} \alpha 9 \mathrm{nAChR}$ cDNA cloned into pGEMHE and transformed into NEB 10-beta high efficiency competent $E$ coli. The result shows that the 18cycle PCR amplification of vector and insert produced many more colonies than the 12-cycle and 24-cycle amplifications $(31,302,43$ colonies for 12, 18, and 24 cycles respectively). For incubation duration in $D p n \mathrm{I}$ digestion, 1, 2, and 4 hours of incubation had similar colony production efficiency with colony numbers of 281, 263, and 285 for 1, 2, and 4 hour-incubations, respectively). However, shorter incubation time is not recommended. In our QuickChange mutagenesis experiment, 30 min incubation in DpnI digestion could not 


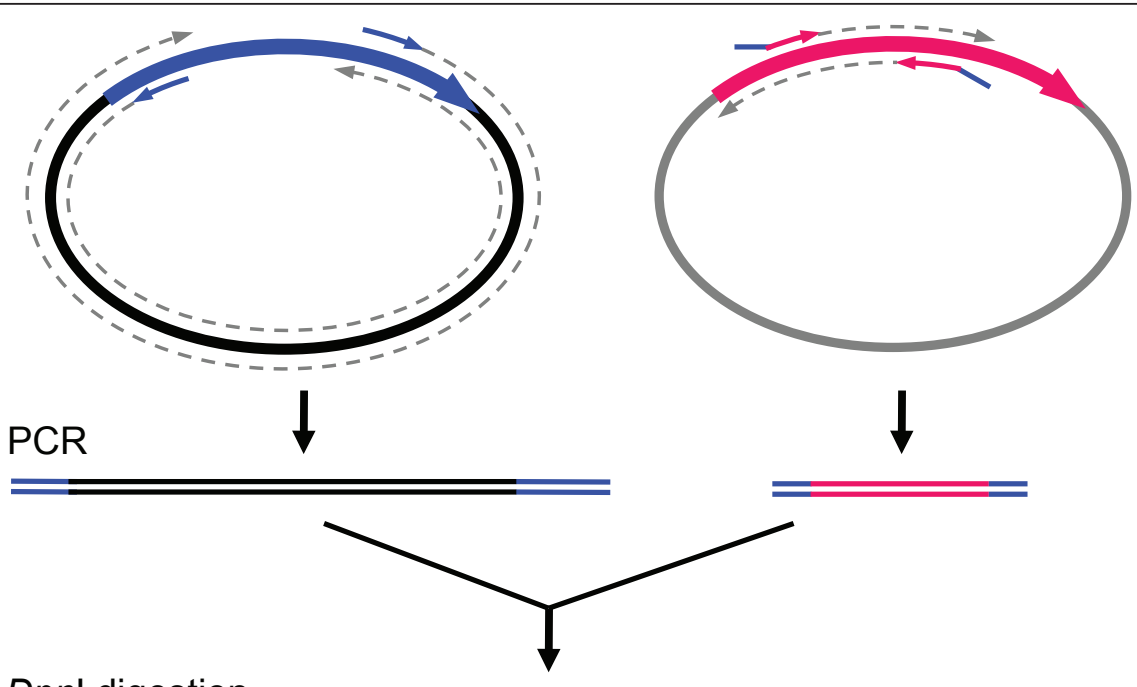

Dpnl digestion
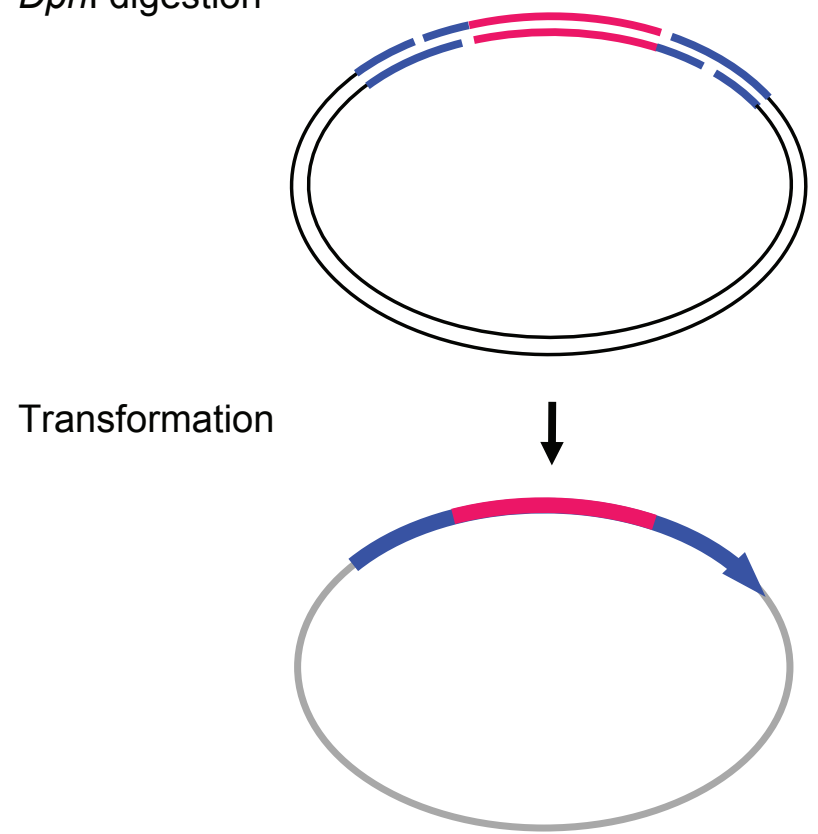

Figure 2 Chimera construction or insertion. Top left: PCR amplification of both ends of the parent cDNA along with the vector. For chimera construction, one fragment of the cDNA needs to be replaced. Thus, the forward primer is immediately downstream of the fragment to be substituted, and the reverse primer will be immediately upstream of the fragment to be substituted. For insertion, however, two primers will be next to each other without skipping a single base. Top right: Insert amplification of the equivalent region of a homologous gene (cDNA) for chimera construction. However, for insertion of a cDNA encoding a full length protein, such as green fluorescent protein, the insert amplification will cover the entire cDNA. The remaining procedure is the same as in Figure 1.

adequately remove the wild-type template background and resulted in a significantly higher fraction of wild type constructs.

In addition, the PCR-based method could produce some unusual constructs. In our $>10$-year practice with the QuickChange site-directed mutagenesis, we only encountered an unusual recombination once. With our FastCloning method in the past few months, we have not seen any unusual construct yet. Thus, if our cloning method produces unusual constructs, their occurrence must be very low. Figure 4D is an example of using restriction digestion to screen clones to exclude unusual constructs. It would be a good practice to perform such a screening for all resulting clones before DNA sequencing. With the optimized cloning conditions (Phusion DNA polymerase, 1:1 vector/insert ratio, 18 cycle PCR amplification, $2 \mu \mathrm{l}$ of vector-insert mix for transformation, and 1 hour $D p n I$ digestion), we have also successfully cloned other cDNAs into the target vectors, and obtained positive clones at efficiency ranging from $43 \%$ 


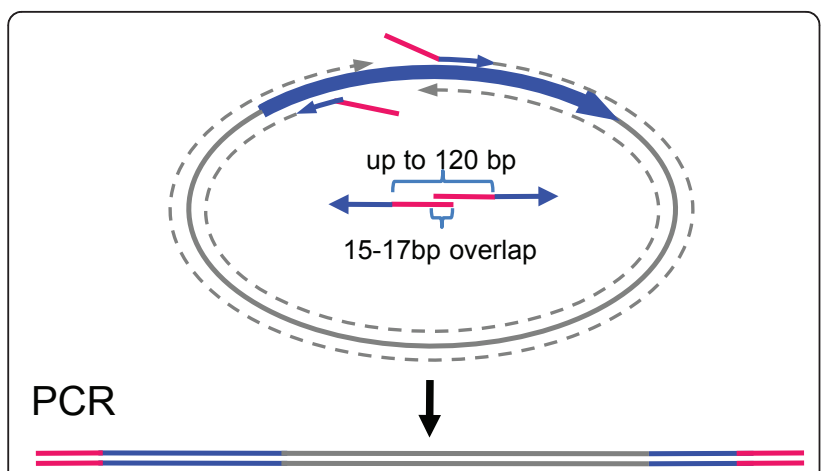

Dpnl digestion $\quad \downarrow$
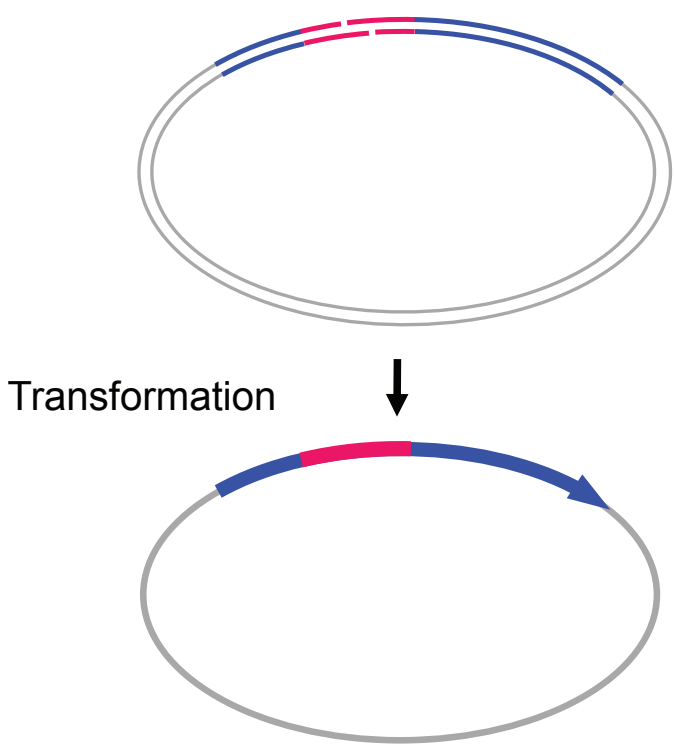

Figure $\mathbf{3}$ Chimera construction or insertion with a short DNA fragment. To replace a short stretch of DNA, such as a DNA fragment encoding a transmembrane region of a nAChR subunit for chimera construction, or to insert a short tag, such as FLAG-tag, into some part of a protein, amplification of the insert is not necessary. In this case, the insert can be directly included in two primers for single PCR amplification of the CDNA along with the vector. The forward primer starts immediately downstream of the insertion site and has a tail with the $3^{\prime}$ part of the insert. The reverse primer starts immediately upstream of the insertion site and has a tail with $5^{\prime}$ part of the insert. Two primers only require $\sim 16$-base overlap. Thus, for a 120 bp insertion, each primer needs to have a 68-base tail for insertion and $\sim 17-22$ bases for annealing (depending on the GC content). The total length of each primer will be about $85-90$ bases. Dpnl digestion and transformation are the same as in Figure 1.

to $100 \%$, with an overall efficiency $>70 \%$. The results further validate the versatility and reliability of this new technique.

Because the PCR amplification of vector can be controlled by primers to exact positions, our FastCloning method is truly sequence-independent. Thus, one can put an insert to any position and in any frame. This feature, although with only a small modification of standard cloning protocol, makes it easy to construct cDNAs for fusion proteins or chimeras. Furthermore, a minor variation of this technique can be applied for insertion of a short DNA fragment directly from two relative long primers for PCR amplification of a cDNA along with its vector. As a proof of principle, we have successfully created human $\mathrm{nAChR}$ cDNAs encoding $\beta 2-\beta 4$ chimeric subunit proteins with $C$-terminal domain swap between the $\beta 2$ and $\beta 4$ subunits. We have also successfully inserted DNA fragments into cDNAs using two long primers with a 16-base overlapping region to directly amplify the cDNA. Figure 5 is an example of using a synthesized insert (99 bp), a DNA sequence encoding a fragment of an acetylcholine binding protein (AChBP), to replace a DNA fragment in the cDNA of the human $\alpha 7 \mathrm{nAChR}$ for chimera construction. Note that each primer contains only slightly more than half of the insert. The primer pairs have a 16-base overlap region. Using similar method, we have successfully replaced other four DNA fragments $(105 \mathrm{bp}, 90 \mathrm{bp}$, $69 \mathrm{pb}$, and $87 \mathrm{pb}$ ) in human $\alpha 7 \mathrm{nAChR}$ with synthetic AChBP fragments and swapped the DNA sequences (66 bp) encoding the second transmembrane domains of human $\alpha 4$ and $\beta 2$ nAChR subunits.

Making multiple mutations in a stretch of DNA is equivalent to making short insertions. Figure 6 is an example of 8 amino acid substitutions ( 8 arginines to 8 glutamines) with multiple nucleotide mutations spanning a 13codon region in the cDNA encoding human $\mathrm{nAChR} \beta 2$ subunit. With this relatively short primer pair (45-base forward primer and 46-base reverse primer), we have successfully obtained this multiple mutations spanning a 13-codon stretch in the cDNA. In addition, we have successfully made a quintuple mutant ( 5 consecutive prolines mutated to 5 alanines) of human $5-\mathrm{HT}_{3} \mathrm{~A}$ receptor subunit with a 31-base forward primer and 35-base reverse primer.

It is important to be aware that with PCR-based cloning, the synthesized primers may not be completely uniform with correct sequences. Random single-base deletions, mutations, or insertions can occur in a small fraction of primers, which result in unwanted deletion/mutation/ insertion in a small fraction $(<5 \%)$ of clones. This is especially true for short insertion with long primers. Thus, sequencing across the primer region is required for ultimate confirmation of each clone. If random mutation happens in one clone, picking up another clone often solve the problem. The random mutation out of primer region is rare with high fidelity DNA polymerases. However, DNA sequencing of the entire coding region is still necessary. In the past 8 months, we have successfully used our new cloning method to obtain 21 constructs. Among the 63 sequenced clones, we found 2 deletions in 2 chimeras constructed with long primer pairs, but found no mutations in the entire regions of all constructs beyond 


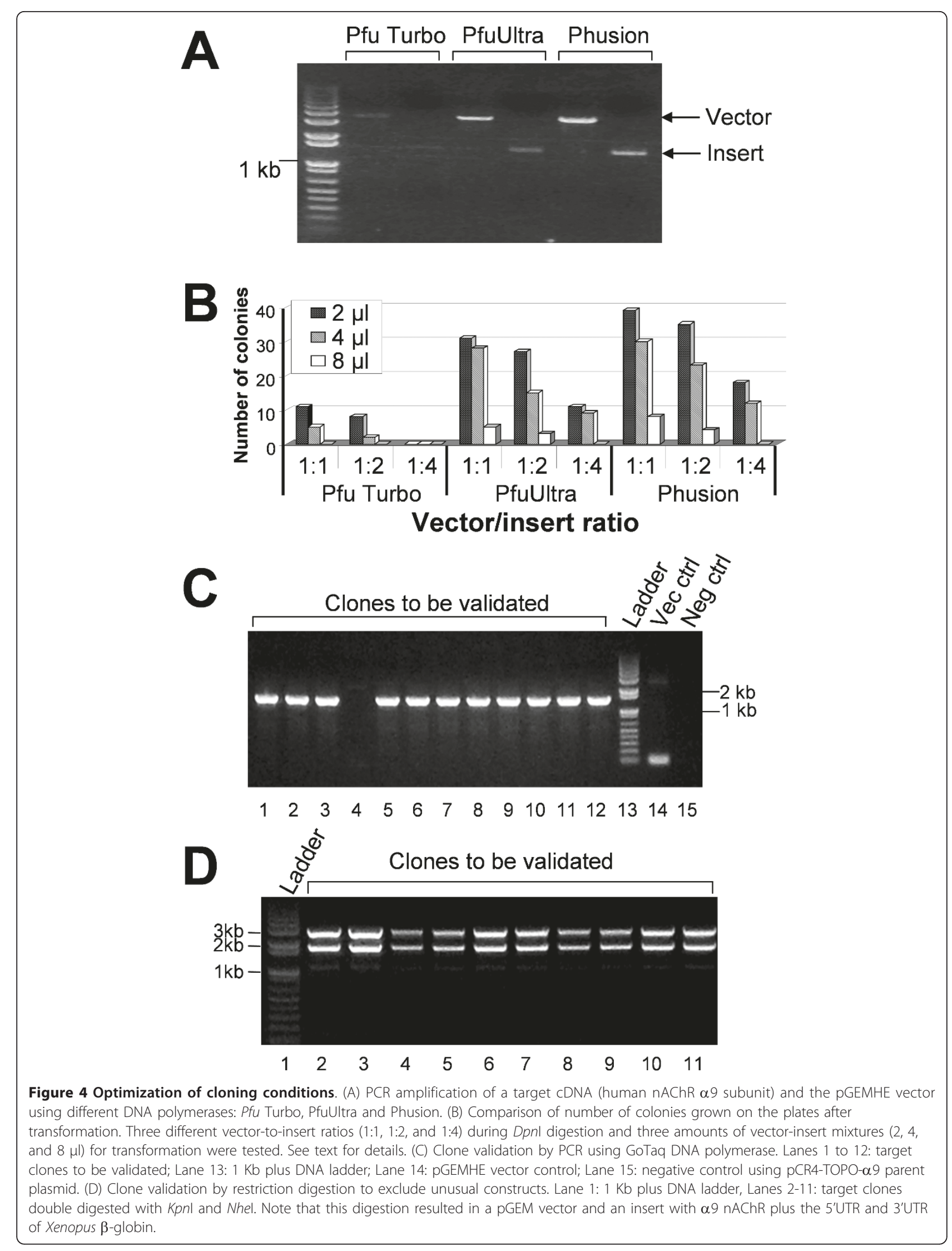




\section{A \\ Wild-type human nAChR $\alpha 7$ \\ CTGCACGTGTCCCTGCAAGGCGAGTTCCAGAGGAAGCTTTACAAGGAGCTGGTCAAGAACTACAATCCCTTGGAGAGGCCC GACGTGCACAGGGACGTTCCGCTCAAGGTCTCCTTCGAAATGTTCCTCGACCAGTTCTTGATGTTAGGGAACCTCTCCGGG $\begin{array}{llllllllllllllllllllllllllll}\text { L } & H & \text { V } & \text { S } & \text { L } & \text { Q } & G & \mathbf{E} & \mathbf{F} & \mathbf{Q} & \mathbf{R} & \mathrm{K} & \mathrm{L} & \mathrm{Y} & \mathrm{K} & \mathrm{E} & \mathrm{L} & \mathrm{V} & \mathrm{K} & \mathrm{N} & \mathrm{Y} & \mathrm{N} & \mathrm{P} & \mathrm{L} & \mathrm{E} & \mathrm{R} & \mathrm{P}\end{array}$ GTGGCCAATGACTCGCAACCACTCACCGTCTACTTCTCCCTGAGCCTCCTGCAGATCATGGACGTGGATGAGAAGAACCAA CACCGGTTACTGAGCGTTGGTGAGTGGCAGATGAAGAGGGACTCGGAGGACGTCTAGTACCTGCACCTACTCTTCTTGGTT $\begin{array}{lllllllllllllllllllllllllll}\mathrm{V} & \mathrm{A} & \mathbf{N} & \mathrm{D} & \mathrm{S} & \mathrm{Q} & \mathrm{P} & \mathrm{L} & \mathrm{T} & \mathrm{V} & \mathrm{Y} & \mathrm{F} & \mathrm{S} & \mathrm{L} & \mathrm{S} & \mathrm{L} & \mathrm{L} & \mathbf{Q} & \mathrm{I} & \mathbf{M} & \mathrm{D} & \mathrm{V} & \mathrm{D} & \mathbf{E} & \mathrm{K} & \mathbf{N} & \mathbf{Q}\end{array}$ \\ B \\ AChBP QANLMRLKSDLFNRSPMYPGPTKDD--PLTVTLGFTLQDIVKVDSSTNEV nAChR 7 VSLQGEFQRKLYKELVKNYNPLERPVANDSQPLTVYFSLSLLQIMDVDEKNQVL \\ 0 \\ AChR $27-A C h B P$ chimera (Black: nAChRa7 sequence, Red: AChBP sequence) \\ ССTGCAAGGCGAGTTCCAGATGAGACTGAAGAGCGACCTGTTCAACAGAAGCCCCATGTACCCCGGCCCCACCAAGGAC GGACGTTCCGCTCAAGGTCTACTCTGACTTCTCGCTGGACAAGTTGTCTTCGGGGTACATGGGGCCGGGGTGGTTCCTG

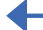

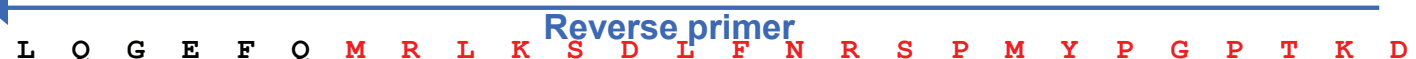 \\ Forward primer

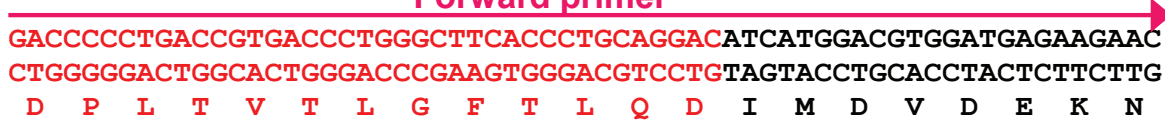

Figure 5 An example of chimera construction with a short (105 bp) DNA fragment replaced by a synthesized insert of 99 bp (included in two primers). (A) A region of $\alpha 7 \mathrm{nAChR}$ subunit sequence with a $105 \mathrm{bp}$ fragment (blue) to be replaced. (B) Amino acid

sequence alignment of human $\alpha 7 \mathrm{nAChR}$ and corresponding region of Aplysia californica AChBP. Segments to be replaced are colored with blue (35 codons) for $\alpha 7$ nAChR sequence and red (33 codons) for AChBP sequence. (C) Target chimera construct with the human nAChR $\alpha 7$ subunit sequence (black) and a substituted 99 bp DNA fragment (red) from the mammalian codon-optimized homologous sequence of the AChBP. Two colored arrows indicate two primers with a 15 bp overlapping region (highlighted). Note that the entire insert of the 99 bp fragment is included in the two primers. Thus, there is no need to amplify the insert. The length of each primer is 81 bases for the forward primer and 76 bases for the reverse primer.

\section{A}

Wild-type nAChRß2 :

CCACGCCATCATTGCGCCCGTCAGCGCCTGCGCCTGCGGCGACGCCAGCGTGAGCGCGAGGGCGCTGGAGCCCTC $\begin{array}{lllllllllllllllllllllllll}\mathrm{P} & \mathrm{R} & \mathrm{H} & \mathrm{H} & \mathrm{C} & \mathrm{A} & \mathbf{R} & \mathrm{Q} & \mathbf{R} & \mathrm{L} & \mathbf{R} & \mathrm{L} & \mathbf{R} & \mathbf{R} & \mathbf{R} & \mathrm{Q} & \mathbf{R} & \mathrm{E} & \mathbf{R} & \mathrm{E} & \mathrm{G} & \mathrm{A} & \mathrm{G} & \mathrm{A} & \mathrm{L}\end{array}$

nAChRß2-8Rto8Q mutant:

CCACGCCATCATTGCGCCC AA CAGCAGCTGCAACTGCAGCAACAGCAGCAGGAGCAAGAGGGCGCTGGAGCCCTC

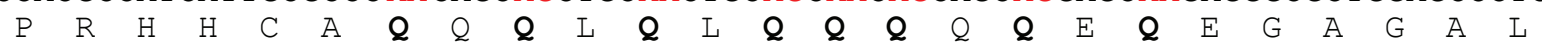

B

Primer pair to generate the $\mathrm{nAChR} \beta 2-8 \mathrm{Rto8Q}$ mutant:

Forward primer

CAACTGCAGCAACAGCAGCAGGAGCAAGAGGGCGCTGGAGCCCTC GGTGCGGTAGTAACGCGGG TTGTCGTCGACGTTGACGTCGTTGTCG

\section{Reverse primer}

Figure 6 An example of making multiple mutations across a wide region. (A) A fragment of the human nAChR $\alpha 2$ subunit cDNA with 8 arginine ( $R$, in bold) codons (top) to be substituted by glutamine $(\mathrm{Q})$ codons (bottom). Mutated nucleotides are indicated in red. (B) Actual forward (45-base) and reverse (46-base) primers with 16 bp overlapping in their $5^{\prime}$ ends. 
primers. Finally, the cloning efficiency of our FastCloning method is high. Of the 21 cloning constructs, we obtained 19 desired constructs in single runs. In 2 experiments, we needed to repeat the procedure to get the final clones. With only one additional construct, we have not obtained final clones after two attempts.

\section{Conclusion}

We have developed a highly simplified and robust PCR cloning technique termed FastCloning. The new technique eliminates the need of PCR purification/gel purification kit and cloning kit. It is ligation-independent and does not require specific sequence in the vector. Thus, one can insert a DNA fragment into a vector at any desired position without considering restriction sites. This feature also makes it extremely easy to make constructs for fusion proteins and chimeras. In addition, it can be used to make short insertions and multiple mutations spanning a wide region (up to $120 \mathrm{bp}$ ) in a cDNA. Finally, it is a highly efficient and reproducible method.

\section{List of abbreviations}

PCR: polymerase chain reaction; nAChR: nicotinic acetylcholine receptor; AChBP: acetylcholine binding protein; CHRNA9: gene name of the human nAChR a9 subunit; CHRNA4: gene name of the human nAChR a4 subunit; CHRNB2: gene name of the human $n A C h R ~ B 2$ subunit; $5-\mathrm{HT}_{3} \mathrm{~A}$ : serotonin receptor type 3 A subunit; HTR3A: the gene name for human $5-\mathrm{HT}_{3} \mathrm{~A}$ receptor subunit; ECSM2: endothelial cell specific molecule 2 (also named ECSCR: endothelial cell-specific chemotaxis regulator); AChBP: acetylcholine binding protein; Akt3v1/v2: protein kinase B gamma variant 1 or 2; ZACN: gene name of the human zinc activated cation channel

\section{Acknowledgements}

We thank Dr. Alan Gibson from the Barrow Neurological Institute for proofreading the manuscript. This work was supported by National Institute of Health (R01GM085237, to YC) and Barrow Neurological Foundation (to YC). This paper is subject to the NIH Public Access Policy.

\section{Author details}

'Division of Neurobiology, Barrow Neurological Institute, St. Joseph's Hospital and Medical Center, Phoenix, AZ, USA. ${ }^{2}$ Department of Obstetrics and Gynecology; St. Joseph's Hospital and Medical Center, Phoenix, AZ, USA. ${ }^{3}$ Department of Genetics and Cell Biology, Guangzhou Medical University, Guangzhou, China.

\section{Authors' contributions}

$Y C$ designed the experiment and most primers, and wrote the manuscript. $\mathrm{CL}$ performed cloning of human a9 and $\beta 2 \mathrm{nAChR}$ subunits into pGEMHE vector, and optimized experimental conditions. He also cloned Akt3v1 or Akt3v2 into pLXSN vector and made a multiple mutation (8 amino acid residue substitutions spanning 13 codons) and several chimeras. AW performed cloning of human 5-HT3A receptor subunit and APBB1 into PGEMHE vector and made a quintuple mutant (5 consecutive mutations) in human 5-HT3A subunit. BS performed cloning of human a4 nicotinic receptor subunit and ECSM2 into pGEMHE vector. $J \mathrm{~L}$ and $\mathrm{YH}$ designed primers for ECSM2 and cloned the ECSM2 into P3xFLAG-CMV-14 vector. YH also contributed to manuscript writing and revision. All authors read and approved the final manuscript.

Received: 3 June 2011 Accepted: 12 October 2011 Published: 12 October 2011

\section{References}

1. Sambrook J, Russell D: Molecular Cloning: A Laboratory Manual. Cold Spring Harbor Laboratory Press, New York; 2001.

2. Marchuk D, Drumm M, Saulino A, Collins F: Construction of T-vectors, a rapid and general system for direct cloning of unmodified PCR products. Nucleic Acids Research 1991, 19:1154.

3. Aslanidis C, de Jong P: Ligation-independent cloning of PCR products (LIC-PCR). Nucleic Acids Research 1990, 18:6069-6074.

4. Haun R, Serventi I, Moss J: Rapid, reliable ligation-independent cloning of PCR products using modified plasmid vectors. BioTechniques 1992, 13:515-518.

5. Walhout A, Temple G, Brasch M, Hartley J, Lorson M, van den Heuvel S, Vidal M: GATEWAY recombinational cloning: application to the cloning of large numbers of open reading frames or ORFeomes. Methods in Enzymology 2000, 328:575-592.

6. Zuo P, Rabie B: One-step DNA fragment assembly and circularization for gene cloning. Current Issues in Molecular Biology 2009, 12:11-16.

7. Bryksin A, Matsumura I: Overlap extension PCR cloning: a simple and reliable way to create recombinant plasmids. BioTechniques 2010, 48:463-465.

8. Yang J, Zhang Z, Zhang X, Luo Q: A ligation-independent cloning method using nicking DNA endonuclease. BioTechniques 2010, 49:817-821.

9. Liman E, Tytgat J, Hess P: Subunit stoichiometry of a mammalian $\mathrm{K}^{+}$ channel determined by construction of multimeric cDNAs. Neuron 1992 9:861-871.

doi:10.1186/1472-6750-11-92

Cite this article as: Li et al:: FastCloning: a highly simplified, purificationfree, sequence- and ligation-independent PCR cloning method. BMC Biotechnology 2011 11:92.

\section{Submit your next manuscript to BioMed Central and take full advantage of:}

- Convenient online submission

- Thorough peer review

- No space constraints or color figure charges

- Immediate publication on acceptance

- Inclusion in PubMed, CAS, Scopus and Google Scholar

- Research which is freely available for redistribution 